Any change from previous or any problems detected by the apps mean stopping the ethambutol and urgent referral to ophthalmology for formal testing.

Outcomes In six months of using the apps, sixteen patients on ethambutol have had regular testing. Two patients have had changes in vision picked up by the apps. One patient's formal eye testing showed no change. The other showed objective change in acuity and colour vision. Without using the apps, these changes may not have been picked up for several more weeks reducing the likelihood of reversibility. The apps are straightforward and a questionnaire of the staff trained in their use rated them easy to use.

Conclusion By using the apps, additional ophthalmology appointments are avoided unless needed and problems are potentially detected before the patient notices any change in their vision. Early detection enables ethambutol to be stopped with the aim of reversing any optic neuropathy before it becomes permanent.

\section{P3 THE CHALLENGE OF ESTIMATING TB MORTALITY ACCURATELY: RECONCILING DEATHS REPORTED IN THE TB NOTIFICATION SYSTEM AND THE VITAL REGISTRATION SYSTEM IN ENGLAND AND WALES, 2005-2015}

${ }^{1}$ MK Lalor, ${ }^{1} \mathrm{~T}$ Mohiyuddin, ${ }^{1} \mathrm{~T}$ Uddin, ${ }^{1} \mathrm{HL}$ Thomas, ${ }^{2} \mathrm{M}$ Lipman, ${ }^{1} \mathrm{CNJ}$ Campbell. ${ }^{1} \mathrm{~TB}$ Section, NIS, Public Health England, London, UK; ${ }^{2}$ Royal Free London NHS Foundation Trust, London, UK

\subsection{6/thoraxjn-2017-210983.145}

Background An accurate estimate of TB mortality is required to monitor progress towards the end TB goal of reducing deaths by $95 \%$ by 2035. In England and Wales (E and W), treatment outcome, including death from all causes, is reported for TB cases notified to the Enhanced Tuberculosis Surveillance system (ETS). The UK Office for National Statistics (ONS) compiles TB mortality statistics from death certificates. We compared data collected in ETS and ONS to inform how best to estimate TB mortality.

Methods TB cases notified in ETS were probabilistically matched to ONS deaths (DONS) between 2005 and 2015 which had ICD-10 codes indicating TB caused or contributed to the death. Deaths reported in ETS (DETS) were identified in DONS to assess if ONS captured all TB deaths. DONS were identified in ETS data to determine if all people dying with TB were notified. Data from ETS and death certificates enabled stratification of deaths into: active TB, TB sequelae and not TB. Risk factors for deaths recorded in only one system were identified with multivariable analysis.

Results In E and W, the number and proportion of DETS (2005: 470 (6.0\%), 2014: $364(5.5 \%)$ ) was lower than the number of DONS (2005: 654, 2014: 587). 57\% of deaths from all causes reported as DETS were recorded as DONS. $53 \%$ of DONS were notified as DETS. In total 9289 deaths were identified in one or both systems: $64 \%$ were active TB, $23 \% \mathrm{~TB}$ sequelae, $7 \%$ were not $\mathrm{TB}$ and in $6 \% \mathrm{~TB}$ was incidental. DETS not recorded in ONS were more likely to be culture and smear negative and diagnosed post-mortem. DONS not notified to ETS were more likely to be female, over 65 years old and born in the UK.

Conclusions Data on TB deaths captured in ETS and ONS differ significantly, suggesting neither system captures all TB deaths. Almost one third of TB deaths recorded by ONS are not active $\mathrm{TB}$, and coding changes in ONS could resolve much of this. Further work, including an audit to determine whether there is under notification of TB or incorrect completion of death certificates or both is needed.

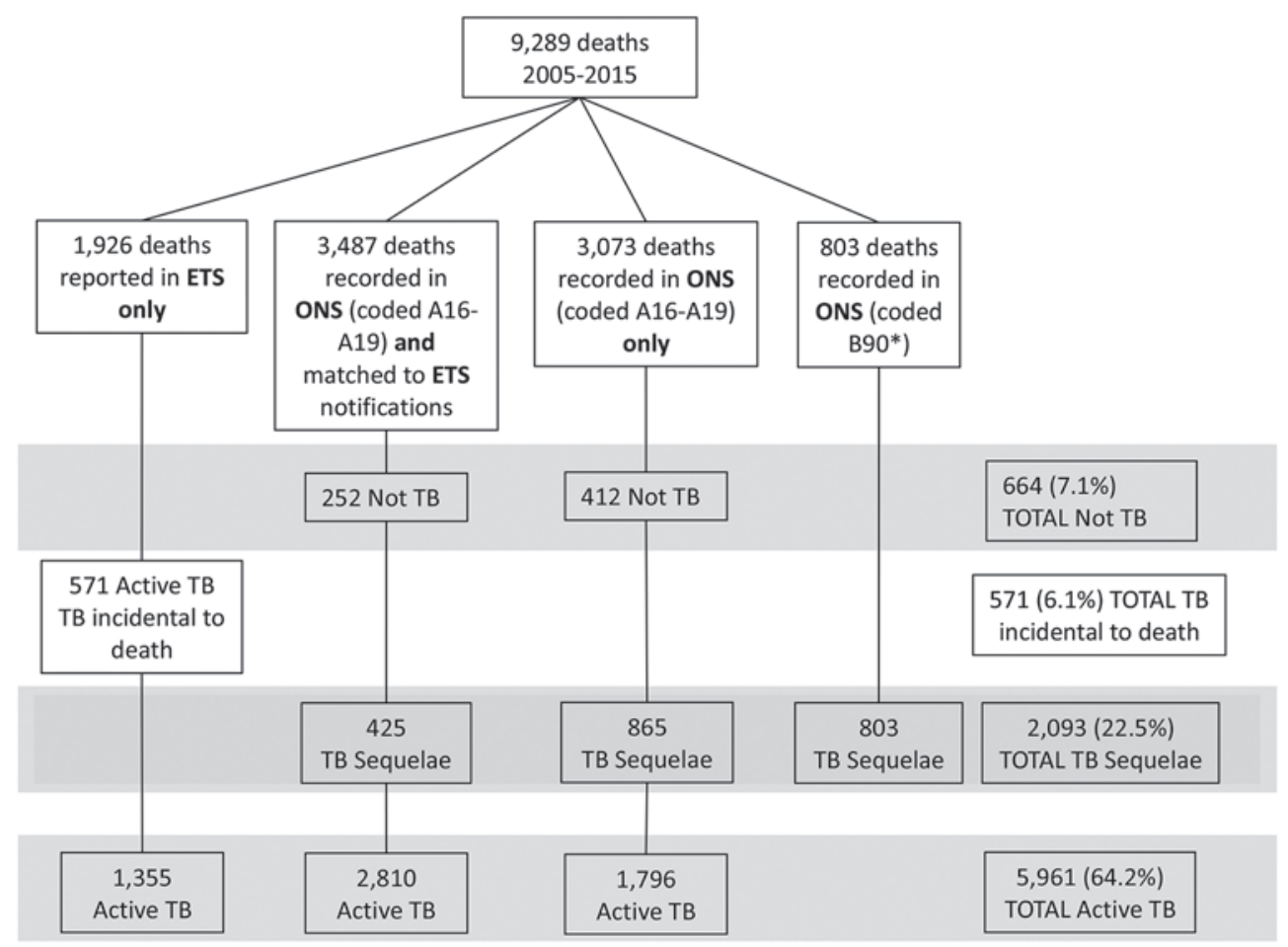

Abstract P3 Figure 1 Flow chart of all "TB deaths" identified in ETS and ONS between 2005 and 2015, including details of which have matched to ETS notifications (cases notified between 2000 and 2015). TB deaths classified into active TB, TB sequelae, not TB and TB incidental to death. 